\title{
Theoretical approaches to bird migration
}

\section{The white stork as a case study}

\author{
A. Kölzsch ${ }^{\mathrm{a}}$ and B. Blasius \\ ICBM, University of Oldenburg, 26111 Oldenburg, Germany
}

\begin{abstract}
Birds are often considered to be one of the best studied groups of organisms. However, only a few investigations have been devoted to a theoretical analysis of avian migration patterns in time and space. This paper is meant to be a first step into this direction. We start by presenting different types of observational data sets that are available and discuss their advantages and disadvantages for use in quantitative analysis of bird movement and migration. Based on ring recovery and satellite telemetry data we perform a statistical analysis of the migratory patterns of the white stork Ciconia ciconia. We find that standard methods from random walk theory can be applied, but have to be carefully interpreted and possibly modified to analyse migration movement data which are dominated by seasonal drift. Our analysis reveals two different modes of movement - fast, directed migration and slow, undirected resting. Furthermore, we present a conceptional network model of avian migration. In our model a number of discrete breeding, resting and wintering habitats are linked by migration in the form of seasonally driven transition probabilities that are described by a unimodal circular function of time. Our study emphasises the need for more rigorous quantitative data analysis and mathematical modelling to gain a better understanding of the dynamic processes of avian migration.
\end{abstract}

\section{Introduction}

Bird migration has fascinated man for a very long time and it has been studied in many aspects and from various perspectives. As a large number of bird species from all parts of the world venture periodic, seasonally driven return migrations of various lengths, one is interested in their routes and the mechanisms driving this phenomenon [1]. There are different forms of bird migration. Some species fly thousands of kilometers between their wintering and breeding areas (long-distance migrants, e.g. the arctic tern Sterna paradisaea), whereas others just move a few hundred kilometers from their breeding grounds to areas of more favourable feeding conditions in the winter. Each migratory bird species has different physiological features that determine its movement behaviour and migration routes. For example, large birds need thermalling to fly long distances [2] which restricts their routes mainly to over-land.

Many species have certain areas on their way that they visit each year for moulting, resting and feeding up. Thus, their migration can be described as a jumping process on a network of more or less discrete habitat patches. Complex networks have been widely used to model spatial processes in the real world [3,4], not least because the discrete space dimension used by this method makes is relatively easy to examine very complex processes and patterns. In spatial ecology network approaches have, for example, been popular for describing metapopulation dynamics on a network of habitat patches or the spread of infectious diseases [5-8]. While the

\footnotetext{
a e-mail: koelzsch@icbm.de
} 
statistics underlying transport processes on complex networks has been intensively studied [3,4], seasonal bird migration implies jumping rates which are not constant, but periodic functions of time. This will change the network and transport characteristics in a fundamental way.

The process of migratory bird movement is not only varying within years, but is exposed to individual variation, between year variability and gradual directional changes during the last years. Due to human mediated habitat destruction and climate change, some birds are altering their migratory routes and timing $[9,10]$. This affects species with different strategies in various ways and may become of concern for their persistence. Another topic that is presently of concern is that the spread of diseases and pathogenic microorganisms has been associated with bird migration $[11,12]$. From an epidemiological point of view it is extremely important to predict the impact of such pathogen dispersal on human populations along migratory routes. These scenarios further emphasise our motivation to characterise migration patterns and to quantitatively describe the process of bird migration.

Up to now only few studies have been devoted to investigate bird migration from a theoretical point of view [13], and if then mostly the physiological mechanisms of optimal flight strategies $[14,15]$ and navigation mechanisms $[16,17]$ were examined in experimental and simulation studies. These aspects of avian migration are thus well under study, even if widely discussed lately [18]. To our knowledge, nobody has yet systematically explored and reproduced the movement of migratory birds in time and space. One noteworth exception is a seminal work by Viswanathan et al. [19]. They investigated the flight distances of albatrosses in the South Atlantic and showed that bird movements can be described as Levy flights. However, this study regarded only foraging movement, while migratory movements which proceed on much larger spatial scales were not included in their analysis.

In a fast changing world it becomes more and more important to understand general ecological processes in detail and determine its underlying courses. By mathematical modelling many phenomena in nature have been systematically examined and one was able to characterise important driving mechanisms (e.g. in epidemiology or ecology $[5,6,8,20,21]$ ). We want to point out that also in bird migration time has come for more abstract and rigorous modelling and data evaluation. There are several challenges that will have to be met, because of the wide ranges of migration routes, their variability between the years and individuals, data deficiencies and the several time dependencies of movement parameters and population characteristics. However, if they are met in a convenient way we may be rewarded with a thorough understanding of avian migratory movement patterns which can be applied in a wide range of disciplines, like habitat management, climate change, optimal flight and epidemiology. This study is meant to be a first step into this direction and our aim is to present some first approaches for a theoretical description of the migratory movement of birds. Another major motivation is to propose this topic to the physics community as an intriguing problem and interesting case study.

We begin with a short review of different kinds of observation techniques that were developed and are applied for qualitatively and quantitatively exploring bird migration in time and space. Special emphasis is given on possible sources of bias and data uncertainty. As an example, we present some preliminary results for the statistical analysis of the movement patterns of the white stork Ciconia ciconia. First, we study the mean square displacement using ring recovery and satellite telemetry data. We find that the migratory dislocation for small time steps is dominated by ballistic movement. For longer time spans, however, the average travel distances are periodic functions of time, which underlines the importance of large-scale seasonal drift and recurrence in the migratory behaviour of white stork. Second, we investigate distributions of turning angles and are able to identify two different flight patterns - a migratory mode characterised by fast, directed movement with small turning angles during the time of migration and a resting mode characterised by slow, undirected movement with strong changes in flight directions especially during the breeding season. Data accuracy is, however, rather poor on small scales, indicating that great care should be taken of any consideration of turning angles. Based on these results we propose a conceptional quantitative model for global avian migration. The model describes a number of discrete breeding, resting and wintering habitats which are connected by fast, directed migratory flight routes. These seasonal transitions of individual birds between different habitat patches are 
described as unimodal circular functions of time. We are able to parametrise the transition probabilities with data from the white stork and find that here satellite telemetry data are relatively well suited. Finally, we discuss applications and possible extensions of our model.

\section{Data for identifying bird migration routes}

\subsection{Ring recovery}

The beginning of the systematic exploration of the routes of migratory birds has been marked by H.C. Mortensen in 1890 putting small metal rings on blackbirds Sturnus vulgaris and other species and receiving recoveries from distant locations. In the following century the ringing of birds spread and has become a highly important tool in exploring the behaviour and ecology of migratory birds all over the world [22]. Many ringing centres were founded that provide standardised rings and ringing techniques and gather recovery data from a diligent crowd of volunteer bird watchers. Before that time bird migration was a rather mysterious phenomenon and theories trying to explain the absence of birds in a given area during a certain time of the year were rather speculative and controversial. The combination of positions of ring recoveries into so called "Atlases" gave a first picture of the spatial distribution of the migration routes of different bird species [1]. Techniques to mark individuals of different species of birds have become more and more refined during the years, e.g. putting large neck or wing bands with numbers or colour rings to be read off from a distance. Thus, birds did not need to be captured for identification any more. As the number of recoveries increased for many species it was also possible to get a crude idea of the temporal course of migration.

However, as recovery data is mainly obtained from amateur bird watchers and the public, the disposition to report observations of ringed birds and also the sampling efforts are not constant $[22,23]$. According to when and in what regions bird watching activity and awareness is high reporting rates and, thus, recovery probabilities differ greatly. In most areas in Africa, for example, reporting rates are suggested to be rather low because of low population densities and rather little awareness of the public of the subject matter of ringed birds and its importance. Therefore, ring data are typically strongly biased, a fact that makes it extremely difficult to quantify the process of migration from such data. Concluding, one can say that ring data has been successfully used in life history and behavioural studies and usually provides a good first idea about migration routes. However, the problems associated with sampling biases may pose serious difficulties when aiming for a quantitatively explicit description of migration patterns.

\subsection{Satellite telemetry}

For many years radio telemetry tags have been used to tackle questions of the behaviour and movement of birds [24]. Individual birds are equipped with a radio transmitter which is sending a signal that can be directly tracked by an observer using a receiver. The transmitters are rather small and obtain positions sufficiently accurate. However, their tracking range is, depending on the vegetation, rather short $(<30 \mathrm{~km})$, and the process of following a bird is considered very labour intensive.

When the ARGOS system (www.argos-system.com) was established in the 1970s for monitoring environmental processes with the help of satellites that orbit our earth, a new tool was available to explore the routes and characteristics of bird migration [25]. Soon, battery powered transmitters were developed. They can be carried by individual birds anywhere on earth, for several months or even years sending signals to a satellite which is calculating the position of the bird and gives back longitude and latitude values to the ARGOS data base. From there researchers can retrieve information about the whereabouts of their studied animals. The technique of satellite telemetry is very powerful, having a wide range, not being very labour intensive and, especially since using solar batteries, even long lived. It has been widely applied in animal movement, orientation and migration studies [26-29], and its potential to e.g. resolve the discussion regarding flight navigation has been emphasised [18]. 
However, there are some drawbacks and problems that one has to consider when analysing satellite telemetry data. The equipment is very costly and the fee for using the satellites high. The transmitters are relatively heavy ( $>10 \mathrm{~g}$, i.e. only applicable to large bird species) and the localisations show large measurement error (especially for smaller transmitters) that is mainly due to inconsistency in the frequency of the signal sent to the satellite [30,31]. Anyway, there is a huge and fast growing amount of satellite telemetry data around, its accuracy is appropriate for analysing long distance movements of migratory animals, and they are highly valuable for determining migration routes of several bird species, especially when compared with the accuracy and time resolution of ring-recapture data. Its accuracy may even be improved by selecting only high quality localisations analysing the additional information that is sent by the satellite [32].

\subsection{New technologies}

Recently, a number of new technologies have become available for animal tracking. Logging devices that receive geographical positions via GPS, for example, are very accurate in positioning, but still rather heavy, and the tags have to be retrieved from the animal to get hold of the stored data [33,34]. Another approach, geolocation via GLS is used to determine the geographical coordinates by measuring ambient light intensity [35]. Compared to GPS the accuracy of GLS is rather poor, being in the range between 30 and $150 \mathrm{~km}$, and the tags also store their data internally [36]. However, GLS tags can be very small (down to $1.5 \mathrm{~g}$ ) so that even small bird species can be studied using them (www.antarctica.ac.uk).

Radar technology has been very promising for studying the behaviour of migrating birds (like e.g. swarming) in a refined area [37]. However, it does not apply to fully explore the migratory routes of long-distance migrants, because immobile radar installations can only track individuals up to a distance of about $300 \mathrm{~km}$. With active radar (e.g. using the cross-band transponder technology, www.earthspan.org) one tried to overcome these limitations and revolutionise radar technology. However, the earthspan project, for example, still faces large problems with detection and battery power. One of the most recent projects in the area of animal tracking is that proposed by Wikelski et al. [38]. They plan to launch a new satellite that can locate low power signals from very small $(<1 \mathrm{~g})$ radio transmitters. If this technique renders feasible localisations obtained from such a satellite will be of extraordinary spatial precision.

To sum up, one can say that there is a wide range of different technologies under development which may revolutionise bird migration mapping in the immediate future. While currently, each method is still facing some problems it is important to be aware of these approaches for future research.

\section{Movement analysis for the white stork}

As discussed above, up to now a large amount of data has been accumulated on the whereabouts of individual birds and is ready for analysis. This is basically the case for ring and satellite telemetry data. In the following, we consider these data types and present a statistical analysis examining the movement of one long-distance migratory bird species, pointing out special features of the processes that govern its movement patterns.

\subsection{Data sets}

As an example of a long distance migrant we have chosen the white stork, because it is one of the best studied bird species in Europe. Some reasons for that are its prominent size and association with civilisation that make observation and captures relatively easy. Much ring recovery data is available for this species and to attach satellite telemetry transponders to several individuals was possible. The white stork is breeding in middle and eastern Europe and migrates either to southern Spain and west Africa or via Israel to eastern and southern Africa. Most individuals 
migrate each year, juveniles usually only partly, but there are also a few nonmigratory white stork individuals that do not leave their breeding grounds in Europe [39]. When migrating the white stork flies only during the day, because it uses thermalling to fly long distances. It does not deposit fat prior to its migration and therefore, in some areas where the white stork rests overnight it may even stay to feed for a few days. Thus, its migration can be described as a stepping-stone process [40].

For the analysis of the movement patterns of the white stork we were able to obtain two different data sets. The first one is based on ring recoveries. The ringing centre "Vogelwarte Hiddensee" has systematically administered bird ringing and compiled recoveries of over 250 bird species in eastern Germany since 1964 [41]. They provided us with ring recovery data of white stork that were ringed and recovered in the eastern part of Germany or that were ringed by different ringing centres and recovered in eastern Germany. Most of the recoveries concentrate on Germany, but several birds have also been observed in their African wintering areas. In total, the data set contains information about 7043 ringed white stork individuals that were at least recaptured one time. Because we are interested in the temporally explicit movement of the birds, we selected only the part of the data set of which the date of the ring recovery had been evaluated to be "correctly known". In Germany some white stork are held in captivity or had been raised by humans and do not migrate. 35 such individuals had to be excluded from the data set, as we wanted to concentrate on strictly migratory birds. The new data set still held recoveries of 5306 individuals; $35.8 \%$ of them were recovered three or more times, one was even recorded 53 times. Of the recovered white stork individuals only $3.8 \%$ have been observed in Africa. A spatial representation of the whereabouts of migratory white storks in the four seasons, as it is obtained from the selected ring data, is shown in Fig. 1. Juveniles (1-3 years old) are included in this data set, because they join the migration of the adults. However, in their first and maybe second summer, these birds possibly stay in the African habitats or just incompletely migrate north, what can be seen in Fig. 1 by the number of recoveries in Africa in June-August.

The second data set that we worked with is of satellite telemetry localisations. During the last 15 years Berthold et al. [27] have attached satellite telemetry transmitters on a large number of individual white storks breeding in Germany and Poland. They were able to obtain several years of movement trajectories for many white stork individuals. One individual has been tracked for more than ten years, revealing much information about its life history and migration ecology [42]. In the light of data quality evaluation, white stork conservation and habitat utilisation the data set has been elaborately analysed [29,31,43]. For our analysis we obtained 3 years (2001-2004) of data from three individual white storks, "Annamarie", "Jonas" and "Prinzesschen". For "Annamarie" it contained 4771, for "Jonas" 1968 and for "Prinzesschen" 5273 relocations. These localisations were spaced relatively evenly over the years, with the exception of poor sampling in May, June and July in the breeding areas. The data sets include individual ring numbers, time, longitude and latitude of each recorded position and several technical and quality measures.

The satellites provide positions of the bird that is equipped with a transmitter quite regularly and rather independently of habitat characteristics and infrastructure. Thus, reporting rates can be assumed constant in time and space. Inaccuracy of the localisations can be detected using frequency stability and other measures provided by ARGOS with each transmitted position [32] and then out-selected. There is naturally a trade off between spatial accuracy and sample size which one has to consider carefully before selecting data for an analysis. Time of recovery is a very accurate measure, and sometimes, especially in more southerly areas, many localisations of the individual bird are available per day. Aware of the problem of poor localisation precision we considered frequency stability measures and chose only data which were evaluated to be accurate to an average distance of about $15 \mathrm{~km}$ [32]. From this set we selected the best position per each day if available for each of the three individuals. Even though the data set was so strongly reduced, we chose to proceed in such a way in order to avoid major problems of spatial inaccuracy on small scales. However we have checked that our main results do not change if we keep all positions per day for analysis. The preprocessed data set consisted of 1538 localisations alltogether that we worked with. Fig. 2 gives a visualisation of the 

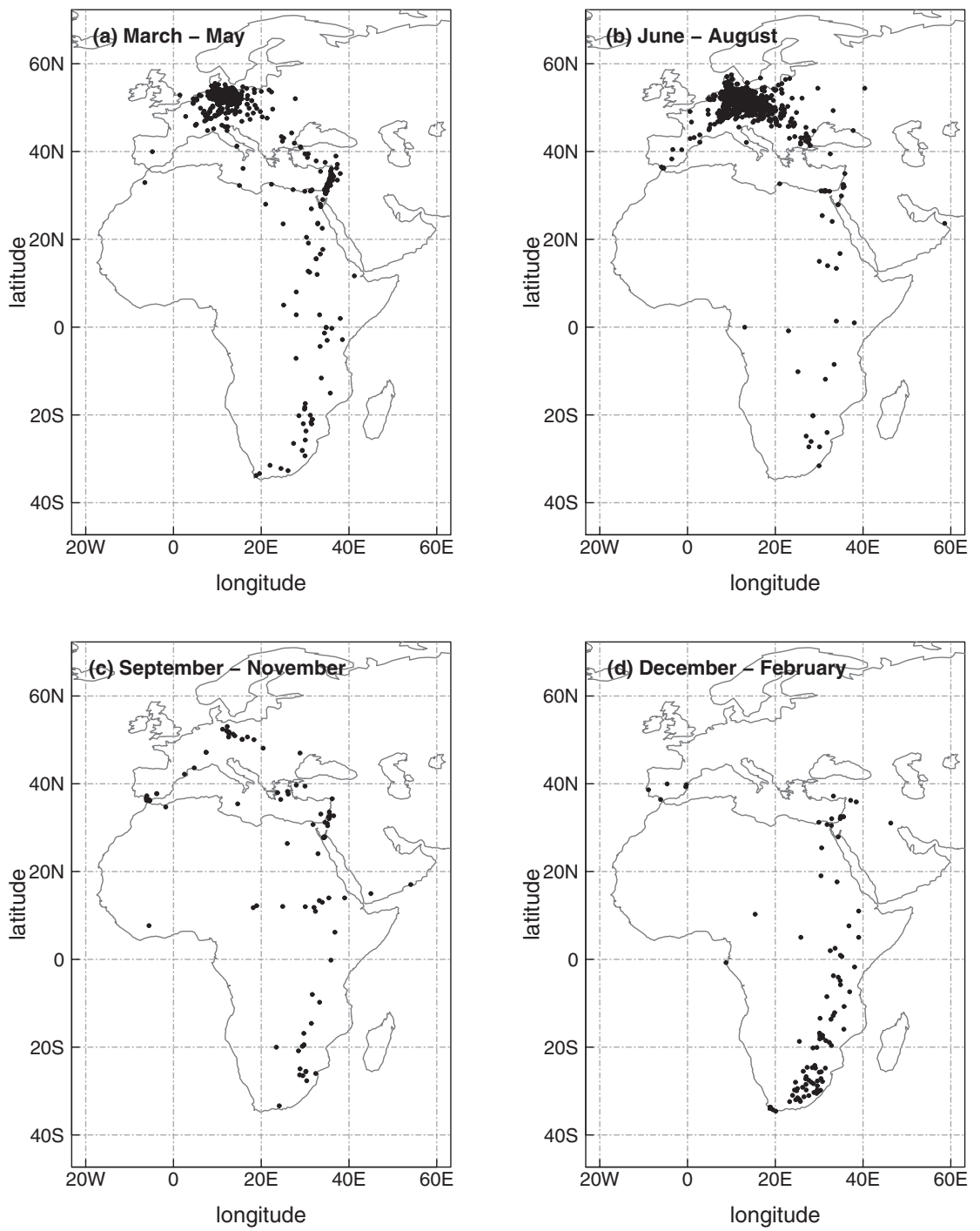

Fig. 1. Migration routes of the white stork, obtained from a subset of ring recovery data of migratory individuals for 1964-2006. Positions of rings recovered in (a) March - May, (b) June - August, (c) September - November and (d) December - February are depicted. Juvenile birds are included here, some of them stay in the African habitats during the breeding period.

movements of the three birds. Note, that even though we have data of only three individuals, they comprise three years each what means that in total our data cover 9 full spring and autumn migration paths. As white stork show individual as well as between year variability in migration routes (differential migration) [42] and because of the selection of three quite differentially migrating individuals we assume that a large amount of migration route variability of the white stork population is captured by our data set. This is supported by a comparison with Fig. 1, where one can see that the migratory routes from ringing and satellite telemetry data agree quite well.

\subsection{Mean square displacement}

One fundamental quantity in random walk theory to characterise the motion of a moving individual is the mean squared displacement. It can be calculated in the same way for both 



Fig. 2. Migratory routes of the white stork, obtained from satellite telemetry data of three individuals from 1 August 2001-31 July 2004. The trajectories represent the migration flight routes of the two east migrating individuals (a) "Annamarie" and (c) "Prinzesschen" and the west migrant (b) "Jonas".

data sets, i.e. the ring recoveries and the satellite telemetry, since both data types yield longitude and latitude coordinates $\left(x\left(t_{i}\right), y\left(t_{i}\right)\right)$ for the geographic position of individual birds at different time instances $t_{i}$. Using these coordinates we analysed the great circle distances

$$
r\left(x_{1}, x_{2}, y_{1}, y_{2}\right)=\left\{\begin{array}{cl}
R \arccos (\operatorname{sign}(s)): & \text { if }|s|>1 \\
R \arccos (s): & \text { else }
\end{array}\right.
$$

where $R=6378.388 \mathrm{~km}$ is the earth radius and $s$ is given by

$$
s=\cos \left(y_{1}\right) \cos \left(x_{1}\right) \cos \left(y_{2}\right) \cos \left(x_{2}\right)+\cos \left(y_{1}\right) \sin \left(x_{1}\right) \cos \left(y_{2}\right) \sin \left(x_{2}\right)+\sin \left(y_{1}\right) \sin \left(y_{2}\right)
$$

with $x_{i}=x\left(t_{i}\right)$ and $y_{i}=y\left(t_{i}\right)$ being the longitude and latitude coordinates in radians of each pair of positions of the same individual. The average distance $d(\Delta t)$ that is travelled in a time span $\Delta t=t_{2}-t_{1}$ is then calculated by the mean square displacement as

$$
d(\Delta t)=\sqrt{\left\langle r^{2}\left(x_{1}, x_{2}, y_{1}, y_{2}\right)\right\rangle} ; \quad \text { for } \quad t_{2}-t_{1}=\Delta t
$$

where the brackets \langle\rangle indicate averaging over all initial positions, $t_{1}$, and all bird individuals in the data set.

For constructing graphical presentations of $d(\Delta t)$ we binned our data into equal 5 day (satellite telemetry data, Figs. 3 and $4(\mathrm{a})$ ) or 30 day (ring recapture data, Fig. 5(a)) time span intervals. We chose these as the minimum interval lengths so that each bin contained a reasonable amount of data (only $1 \%$ of the bins with less than 50 (for telemetry) or 10 (for rings) values of displacements). As can be expected, the number of displacement values calculated from satellite telemetry trajectories decreases for increasing $\Delta t$, ranging from 4328 for $\Delta t \in(5,10]$ days to 14 for $\Delta t \in(1090,1095]$ days. The number of values for different $\Delta t$ in the ring recapture data rather varies depending on the season, 3531 to 207 per month in the breeding period and 89 to 6 values per month during wintering. This variation may somewhat decrease power in the statistics, but sample sizes are still sufficient to allow for interpretation. Furthermore, when varying the bin size between 2 and 50 days for the satellite telemetry data the main characteristics of the plots do not change.

Usually in diffusion problems the average travel distance is an increasing function of the time lag and scales as a power law $d \sim(\Delta t)^{\alpha}$. This relationship can be conveniently depicted in a log-log-plot of $d(\Delta t)$ against all values of $\Delta t$, where the exponent $\alpha$ is obtained as the slope of a least-square fit in the scaling region. This is shown in Fig. 3 for the satellite telemetry data of the three white stork individuals. For small time differences of $\Delta t<6$ months the average 


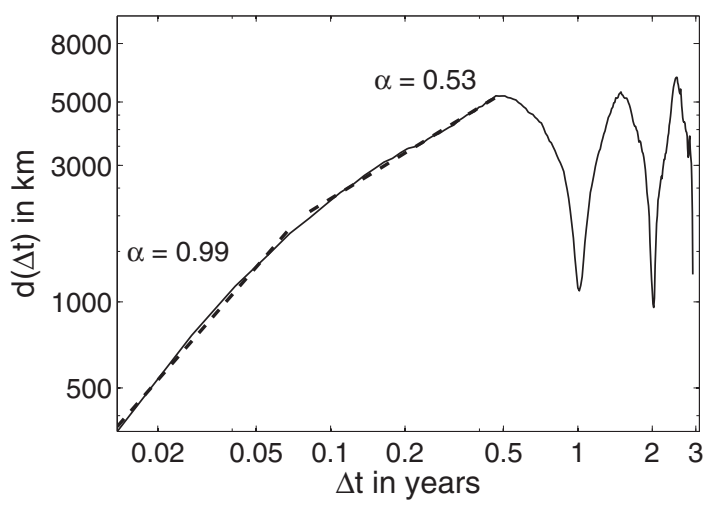

Fig. 3. Average travel distance $d(\Delta t)$ of the white stork on their migratory path obtained from satellite telemetry data, binned into equal 5 day time intervals. This visualisation as a $\log -\log$ plot clearly indicates the superdiffusive character of the migratory movement for small time differences. It may even be split into one part of ballistic $(\alpha \approx 1)$ and another part of diffusive $(\alpha \approx 0.5)$ motion.

travel distance roughly scales with an exponent of $\alpha \approx 0.7$ and the examined motion clearly is superdiffusive. More detailed, for very small time distances of $\Delta t<30$ days the motion is nearly ballistic $(\alpha \approx 1)$, while in the intermediate range of 30 days $<\Delta t<6$ months it may be best described as diffusive $(\alpha \approx 0.5)$. Similar transitions from ballistic to diffusive behaviour are frequently observed in random walks with short term correlations.

Additionally, as can be expected from the seasonally driven character of migration, for larger time scales of $\Delta t>6$ months $d(\Delta t)$ becomes a periodic function of time (Fig. 4(a)). On average each bird returns to its previous position after one year. When aggregating the displacements according to normalised time differences in years, $\Delta \tau=\Delta t \bmod 1$, we can observe this seasonality in form of a hump shaped curve (Figs. 4(b)). Its general form shows that on their migratory path the birds' positions are on average maximally separated by a distance of about $6000 \mathrm{~km}$ at a time difference of about 6 months. This is basically a result of the dominating long distances between the birds' breeding and wintering areas. The slopes of the curve are determined by the speed of migration in spring and autumn, respectively, and the width of the peak's plateau indicates how long the birds stay in their wintering and breeding areas. It is interesting to note the changes of scaling detected in Fig. 3 also in Fig. $4(\mathrm{~b})$ at $\Delta t \approx 1.5$ months and, because of symmetry, at $\Delta t \approx 10.5$ months.

Similar dynamics as for the satellite telemetry data can be deduced from ring recapture data, where $d(\Delta t)$ is obtained from rather sparse trajectories of a very large number of birds over many years (Fig. 5). As we have mentioned above, the ring recovery data base covers a time interval of more than 50 years. This data set contains many recoveries of individual birds with a time interval $\Delta t$ of more than 20 years. However, the frequency of such entries decays for larger time intervals, which results in poor statistics. Therefore, in Fig. 5 we have plotted $d(\Delta t)$ only for time lags up to 10 years. Nevertheless, ring recovery data reflect much larger time spans than the three years of satellite telemetry data that we obtained. In this sense the two data sets can be regarded to be 'orthogonal'. While with satellite telemetry, by averaging over many time instances of only three individual birds, $d(\Delta t)$ is basically calculated as a time average, in the case of the ring recoveries, by averaging over a large number of individuals, it rather corresponds to a sample average.

Even though the ring data fits well into the patterns obtained from satellite telemetry data and the overall form of the average travel distances $d(\Delta t)$ agree in both data sets, there are some marked differences in the shape of the curves. While the rise of $d(\Delta \tau)$ is nearly linear for the satellite telemetry data (Fig. $4(\mathrm{~b})$ ), in the case of the ring recovery data we find abrupt changes at $\Delta t=5$ months and at $\Delta t=8$ months (Fig. 5(b)). We can attribute these discrepancies to the fact that the ring recovery data are not uniformly sampled through all seasons (see also Fig. 1). Individual birds which travel the full distance from Northern Europe to South Africa are most strongly sampled in the wintering and breeding areas at the extreme ends of the migratory path. This results in a sudden peak of $d(\Delta \tau)$ when $\Delta \tau \approx 6$ month. This edge in the 

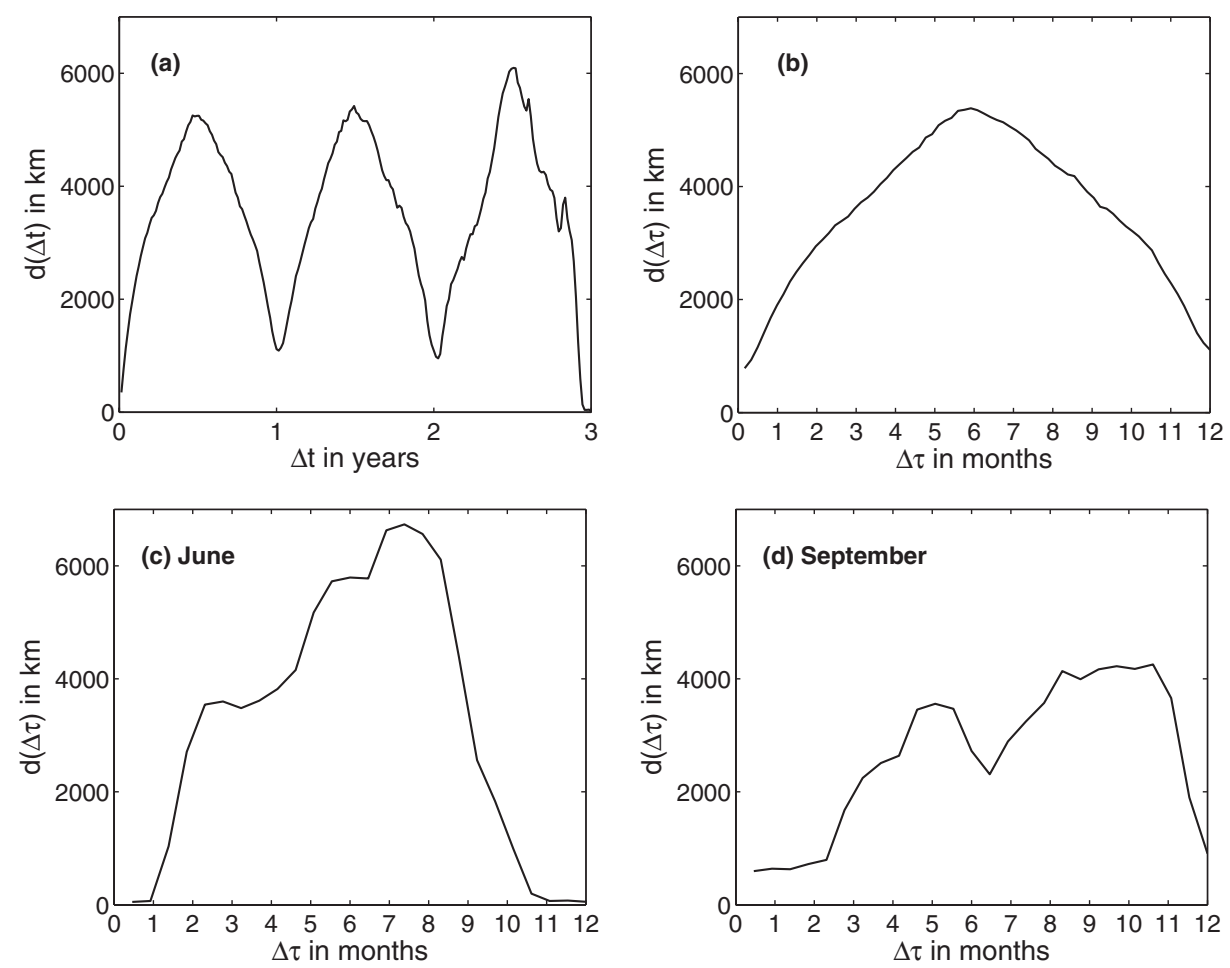

Fig. 4. Average travel distance $d$, from Eq. (3), of the white stork based on satellite telemetry data as a function of (a) the time differences $\Delta t=t_{2}-t_{1}$ and (b) the time differences modulo one year $\Delta \tau$. Similar to Fig. 3 note the change of scaling at $\Delta \tau=1.5$ months and $\Delta \tau=10.5$ months in (b). For further investigation the data are pooled by the displacement starting times $\tau_{1}$ : (c) the middle of the breeding period (18 June-1 July) and (d) the autumn migration period (10 - 23 September). Thus, (c) represents the consecutive displacement of the storks from their breeding area to wintering places and (d) the displacement from some resting site on their autumn migratory route to the wintering grounds, then back, close by and to the breeding areas. The data was binned into equal 5 day time intervals.

curve of typical travel distances is absent in the satellite telemetry data which are uniformly sampled. Thus we believe that in this case the data from satellite telemetry reveal a better picture of the white stork movement patterns.

Interestingly, in both data sets the oscillations of $d(\Delta t)$ are not damped for long time intervals. Instead, the average travel distance always returns to a small value close to zero when $\Delta t$ is a multiple of one year and these minima do not increase with the number of years between two measurement points. This observation confirms the legendary spatial memory and faithfulness to its nesting place of the white stork [44]. In the ring recovery data several birds are located at exactly the same nest, breeding, for many years. The satellite telemetry data underline this notion. The minimum displacements $d_{\min }(\Delta t)$ for $\Delta t$ being multiples of around one year, are of the orders of quality uncertainty of this data set ("Annamarie": $\left\langle d_{\min }(\Delta t \approx k)\right\rangle=272 \mathrm{~m}$, "Jonas": $\left\langle d_{\min }(\Delta t \approx k)\right\rangle=121 \mathrm{~m}$, "Prinzesschen": $\left\langle d_{\min }(\Delta t \approx k)\right\rangle=303 \mathrm{~m}$, for $k=1,2,3$ years). Thus, individual birds come very close to their previous resting, and especially breeding, positions. As shown in Fig. 5 this memory lasts at least 10 years, i.e., the length of our time series. However, also in the full time series including many ring recoveries with time intervals well over 20 years (not plotted) this tendency prevails. Indeed, the longest time interval of recovery in the data base was obtained for a bird that was ringed in Germany as a hatchling and recovered 29 years later only $17 \mathrm{~km}$ from its birth place. This suggests that the spatial memory of white stork individuals extends over the full life span of the birds.

Our analysis has shown that many characteristics of bird migration can be obtained from and are reflected by an inspection of the mean square displacement. However, it also becomes 

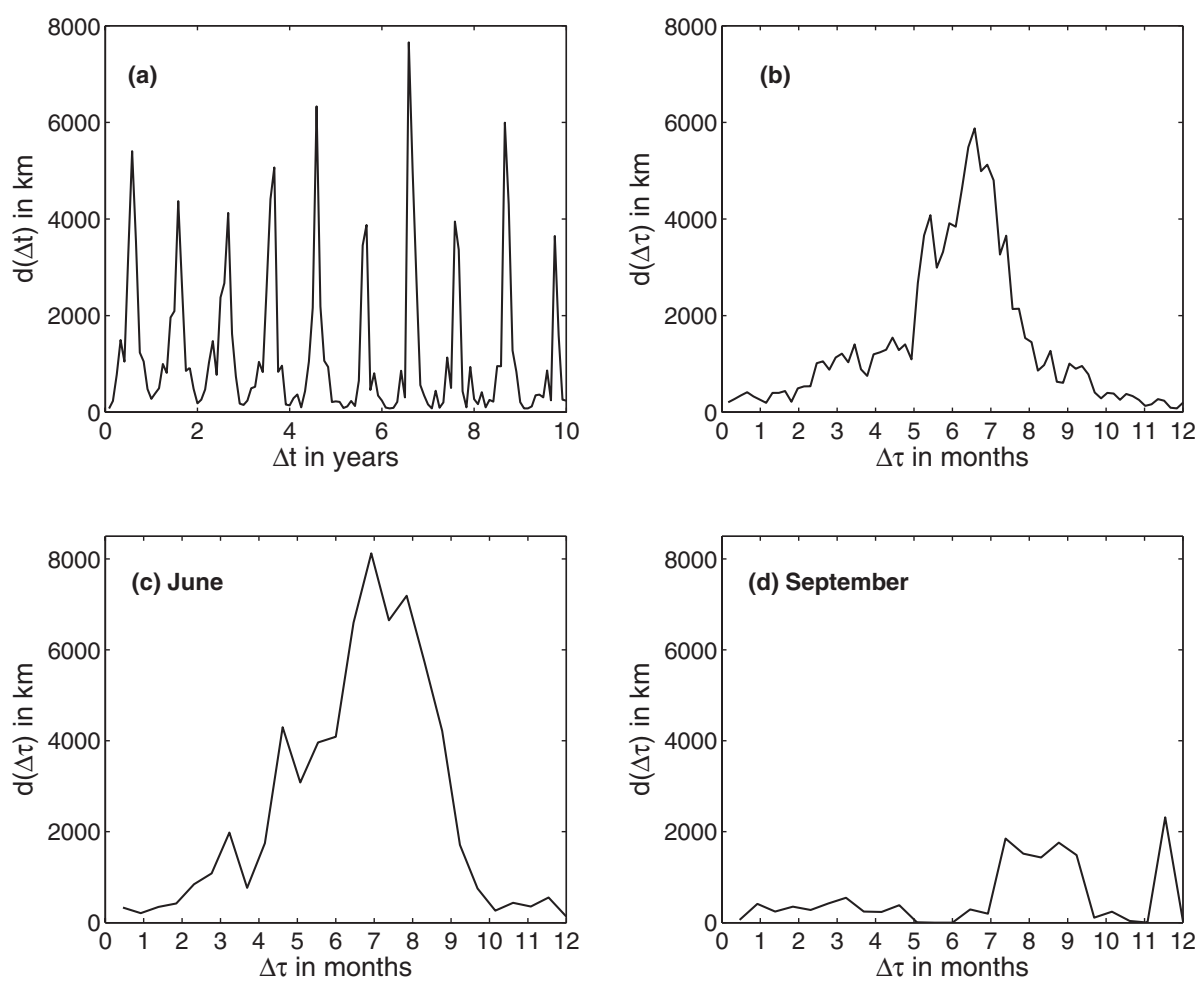

Fig. 5. Average travel distance $d$ of the white stork as in Fig. 4 but based on ring recovery data. The displacements were binned into equal 30 day time intervals for (a), but resolved more finely for the aggregated plots: 5 day intervals for (b) and 14 day intervals for (c) and (d).

clear that in a seasonally driven process like migration such standard techniques from random walk theory should not be applied naively. It is advisable to modify them somewhat and interpret results carefully. A convenient way to obtain a visual impression of the seasonal effect on displacement distances is to split the data according to the starting month of each displacement $d\left(\tau_{1}, \Delta \tau\right)$ (as examples see subplots c and $\mathrm{d}$ in Figs. 4 and 5 ). Here $\tau_{1}$ is the season of the first location of the bird displacement, $\tau_{1}=t_{1} \bmod 1$. In such seasonally pooled presentations the times of breeding and migration can clearly be distinguished, which in this way gives a better indication of how the averaged quantity $d(\Delta \tau)$ is assembled from very different processes during the different stages of bird migration. In Figs. 4(c)/5(c) and $4(\mathrm{~d}) / 5(\mathrm{~d})$ respectively, we contrast such pooled displacement plots for trajectories starting in the breeding area (c) and sometime in the autumn migration (d). In the former ones one sees the difference in migration velocity between the autumn migration (shallowly increasing slope) and the spring migration (steeply decreasing slope), whereas the latter figures more clearly indicate the back-and-forth character of the migratory movement with their bimodal shape.

By comparison of the results for both the ring recapture and satellite telemetry data one obtains insight into the accuracy and quality of the two data types. Additionally, our investigation reveals the advantages to combine such two 'orthogonal' data sets for analysis. While one data set has a very fine resolution over a short time span, the other covers larger time distances at the cost of poor resolution and biased sampling. By combining both data sources we obtain a more complete picture of the migratory movement from two different points of view.

\subsection{Turning angles}

Another important statistics to consider in animal movement analysis is the distribution of turning angles. It indicates the character of the movement, i.e. how directed or curved it is. 


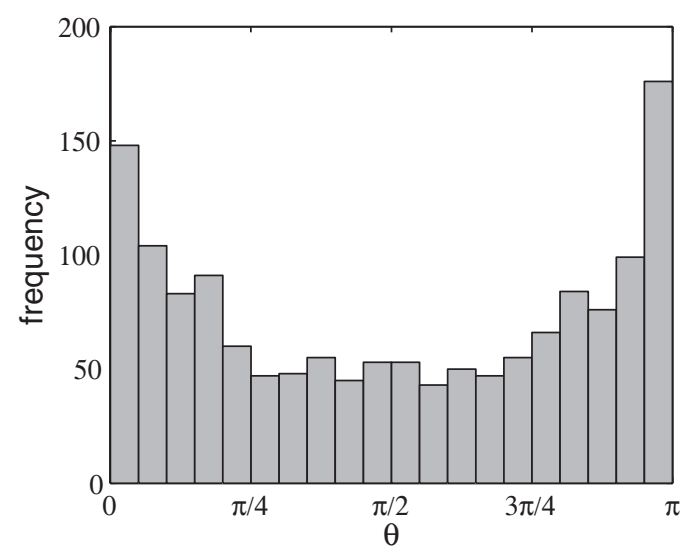

Fig. 6. Histogram $P(\theta)$ of turning angles $\theta$ along the migratory path of the white stork accumulated over the whole year, based on satellite telemetry data.

For migratory birds one would expect that during migration flight is very directed and turning angles are relatively small and that during resting, breeding and wintering turning angles are larger and more or less random in the process of foraging. Analysis of turning angles has been carried out to examine the motion of several aquatic and terrestrial organisms (e.g. daphnia [45], barnacle larvae [46], fish [47], marine mammals [48], ungulates [49], birds [50] and others [51]), usually describing their foraging behaviour or dispersal. However to our knowledge, the distribution of turning angles has not yet been investigated for the year round travel and motion of migratory birds.

Any reasonable measurement of turning angles requires that the time differences between successive positions are small. In our case this is only valid for satellite telemetry data, and therefore we have disregarded ring recovery data for the investigation of turning angles. For the analysis, we selected one best position of each individual per each day that data was available for. From this reduced time series we then selected the triples of positions, whose consecutive time differences were not larger than one week, and calculated a movement direction

$$
\varphi_{i}=\arctan \left(\frac{\Delta y_{i}}{\Delta x_{i}}\right),
$$

where $\Delta y_{i}=\left|y_{i+1}-y_{i}\right|$ and $\Delta x_{i}=\left|x_{i+1}-x_{i}\right|$ describe the differences of geographic latitude and longitude coordinates. Turning angles $\theta_{i}$ are then obtained as the differences of two consecutive movement directions

$$
\theta_{i}=\varphi_{i+1}-\varphi_{i}
$$

The (non-normalised) distribution $P(\theta)$ of turning angles for the migratory movement of the white stork is shown in Fig. 6. It clearly shows a maximum for small turning angles $\theta \approx 0$ and is decaying for larger angles. However unexpectedly, there is a second maximum for large turning angles of $\theta \approx \pi$. Thus, one can suggest that the flight behaviour of migratory birds splits into two characteristic modes. The first mode describes directed flight where the bird changes its direction only little between the days. In contrast, the second mode is characterised by large turning angles of about $180^{\circ}$ corresponding to ongoing reversions of the flight direction.

To obtain more insight into this phenomenon we have resolved to the same approach as in the previous section and have split the histogram according to the starting month $\tau_{1}$. Inspection of the pooled distributions $P\left(\tau_{1}, \theta\right)$ in Fig. 7 reveals that the small turning angles correspond to the typical times of migration (March, August) and that the large turning angles occur mainly during the breeding and wintering seasons. Thus, the cluster of small turning angles results as expected from the directed migration flights in spring and autumn. Similarly, the cluster of large angles can be interpreted in the way that in their breeding and wintering grounds individuals have a central nest or sleeping tree, from where they fly back and forth to a small 

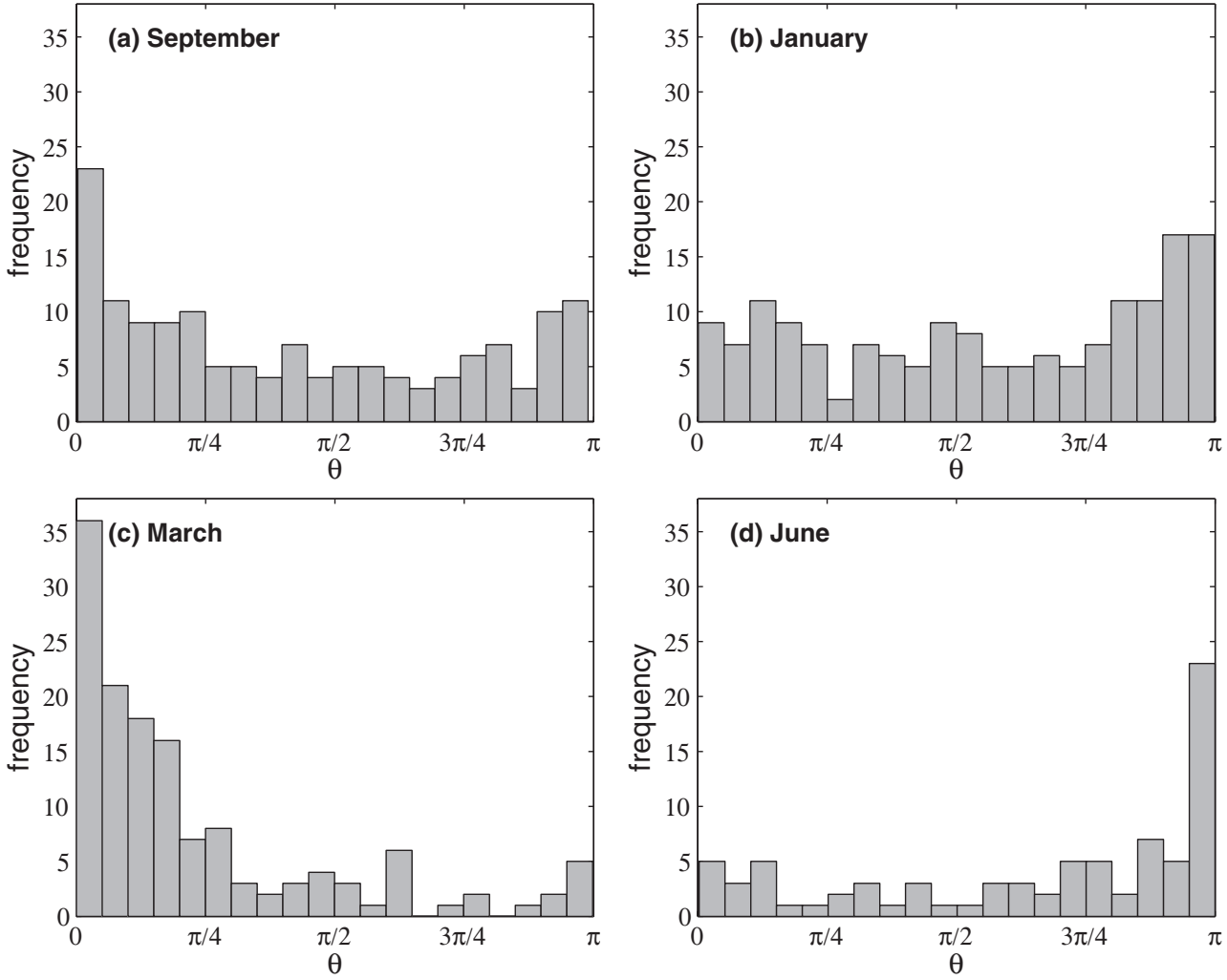

Fig. 7. Pooled distributions of turning angles for the white stork. In contrast to Fig. 6 here the histograms $P(\theta)$ have been split according to the time of the year $\tau$, for (a) September - autumn migration, (b) January - wintering, (c) March - spring migration and (d) June - breeding.

number of foraging areas. To examine this matter in more detail, in Fig. 8 we present a number of two-monthly trajectories for a single white stork and year. Visual inspection of the flight trajectories confirms the existence of these two different migratory flight modes.

We note though that an additional contribution to the cluster of large turning angles might possibly arise from measurement error since the small scale movement in the resting areas contains some localisations of very low accuracy, whose incorporation into the trajectories can produce large spurious turning angles. Indeed, a closer examination of the localisation errors in the breeding and wintering areas (see Figs. 8(b) and 8(d)) shows that the positions at the end of the "spikes" that determine the large turning angles are of rather low accuracy of on average $\pm 15 \mathrm{~km}$, opposed to only $\pm 3 \mathrm{~km}$ for central positions. However, the typical distance between the two extreme ends of a back-and-forth step is larger than $15 \mathrm{~km}$, which makes it unlikely that imprecise measurement can be the sole explanation for the peak around $\theta \approx \pi$. Another phenomenon where large turning angles may occur even during migration is reverse migration, where individual birds reverse their migration direction for a few stages due to some cause that has not yet fully been understood $[1,2]$.

\section{Quantitative modelling of bird migration}

As the result of our movement analysis, a typical path of a migrating bird can be described as an alternating sequence of directed (moving) and undirected (resting) stages. This is consistent with observations in other studies, where the migration of birds has been described as a succession of flying and stopover [15]. In the following we will use these ideas for setting up a conceptional model to describe the seasonal process of migration. 

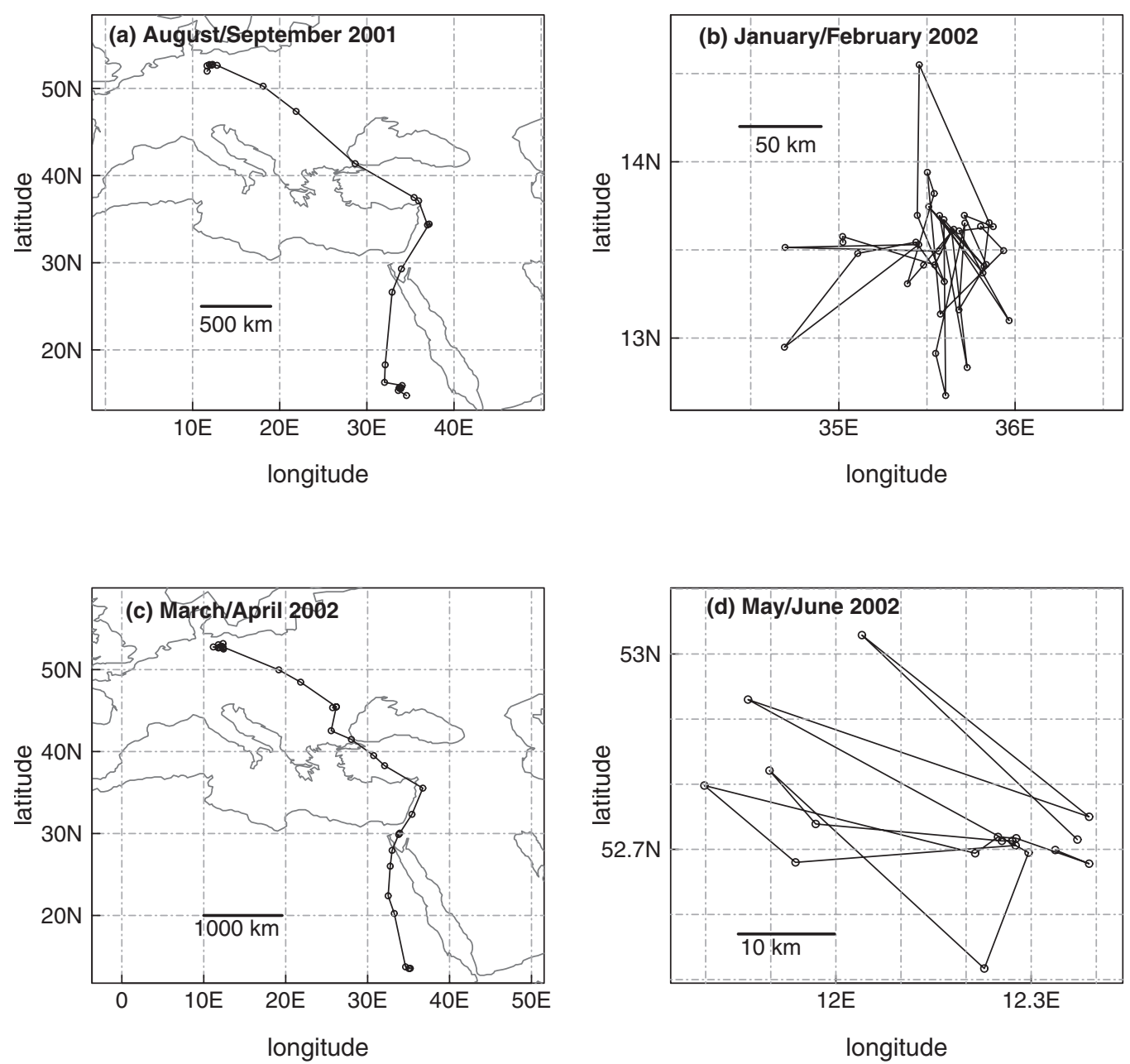

Fig. 8. Selection of typical trajectories of two months along the migratory path of the white stork "Annamarie" for (a) August/September 2001 (autumn migration), (b) January/February 2002 (wintering), (c) March/April 2002 (spring migration), (d) May/June 2002 (breeding).

The main idea of our model is to replace the spatially continuous trajectory of a migrating bird by a jumping process on a network of a number of discrete habitat patches. Thus, the model describes the seasonally driven long-distance migration of birds on a discrete network of $m$ species specific breeding, moulting, resting and wintering places. These local regions, the nodes of the network, are fixed in time and are connected to one another by directed flight routes. Accounting for differential migration we assume that avian migration does not take place only on a linear chain of patches, but instead permit branching on the network of possible flight connections.

Consider the time varying density $n_{i}(t)$ of $N$ birds that are present in area $i=1 \ldots m$ at time $t$. The densities are normalised as

$$
\sum_{i=1}^{m} n_{i}(t)=N .
$$

Thus, we assume that the total number of birds, $N$, stays constant and we neglect population growth during the time scale of our analysis. The dynamics of the migration is described as a 


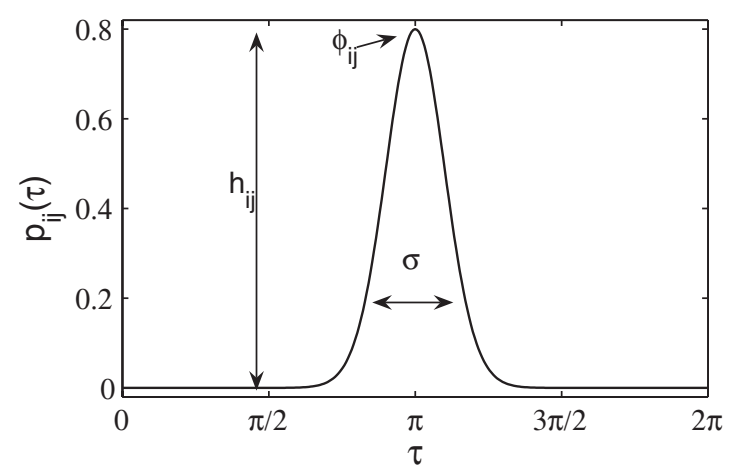

Fig. 9. Typical form of the circular jumping probability function $g(\tau)$, here as a modified form of the von-Mises distribution function, Eq. (9), with parameters $h_{i j}=0.8, \phi_{i j}=\pi$ and $\sigma=0.3$.

Markovian jumping process

$$
n_{i}(t+1)=\sum_{j, j \neq i} p_{i j}(\tau) n_{j}(t)+p_{i i}(\tau) n_{i}(t)
$$

where we assume that the birds jump from patch $j$ to patch $i$ according to certain transition probabilities $p_{i j}$ which do not depend on previous jumping events or bird densities. So, in our model individual birds have no memory and choose the jumping routes every year anew from the same probability distribution. Furthermore, each bird in a given area will continue its migratory path independently of the behaviour of the other birds, thus, we neglect swarming effects.

The novel aspect of our model is that the transition probabilities $p_{i j}$ are not constant within the year, but instead are periodic functions of the season $p_{i j}=p_{i j}(\tau)$ (however, they are assumed to be constant between the years). Thus, the transition probabilities are described by a circular function $g(\tau)$ of the season $\tau$ that quantifies the intensity of birds flying between different patches

$$
p_{i j}(\tau)=g\left(h_{i j}, \phi_{i j}, \sigma_{i j}, \tau\right) .
$$

Each jumping probability $p_{i j}(\tau)$ is characterised by the mean season when jumping takes place (the phase) $\phi_{i j}$, the maximum jumping intensity (the amplitude) $h_{i j}$ and a typical length of the time interval during which jumping occurs (the width) $\sigma_{i j}$. An additional assumption that simplifies the migration jumping process in a reasonable manner is to set the jumping durations equal for all connections of the network $\sigma_{i j}=\sigma=$ const (see Fig. 9). Then each link is characterised by a complex number $z=h \mathrm{e}^{\mathrm{i} \phi}$, which describes the amplitude $h$ and the phase $\phi$ of the transition.

Because it is a canonical distribution to describe unimodular, circular data, we used a generalised von-Mises distribution function as a first approximation of the functional form of $g$

$$
g\left(h_{i j}, \phi_{i j}, \sigma, \tau\right)=h_{i j} \exp \left[\frac{\cos \left(\tau-\phi_{i j}\right)-1}{\sigma^{2}}\right] .
$$

For small values of $\sigma$ this form is nearly a Gaussian with a standard deviation of $\sigma$, while in the limit of large $\sigma$ this approaches a uniform distribution $g(\tau)=h_{i j}$. Furthermore, by using the von-Mises distribution we also assume that the jumping probabilities are symmetrical in time, which may not be the case in real bird migration.

A systematic presentation of the resulting model dynamics will be presented elsewhere. Here we are concerned with the problem of finding an optimal parametrisation in accord with real observational data, i.e. we are interested to estimate the transition rates $p_{i j}(\tau)$ for the migratory movement of the white stork. Data ideal for fitting the described model need to be explicit, accurate and consistent in time and space. As explained above satellite telemetry data, 
(a)

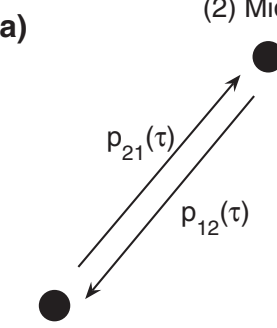

(1) Iberia
(2) Middle Europe

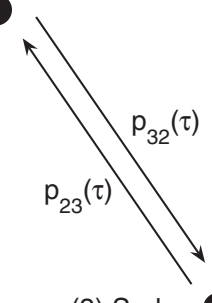

(3) Sudan

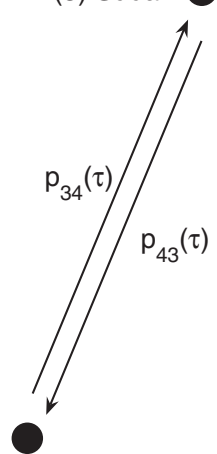

(4) South Africa
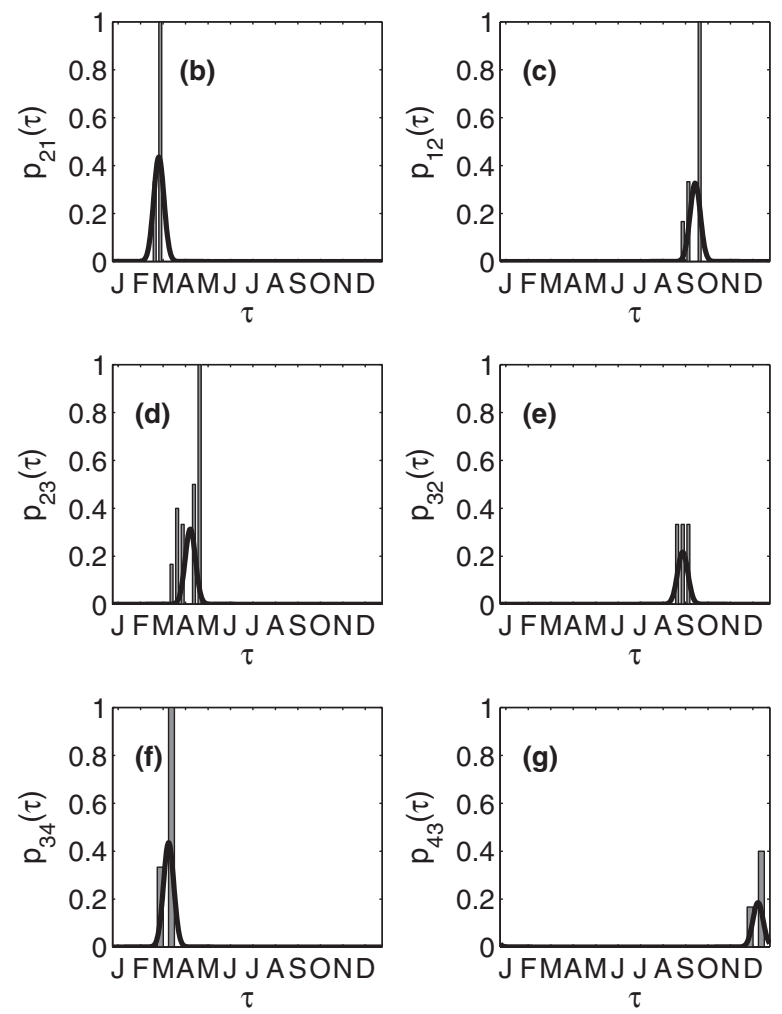

Fig. 10. Network model for white stork migration. The breeding area (2) Middle Europe is connected to the main wintering areas (1) the Iberian Penninsular, (3) the Sudan area and (4) South Africa by seasonally driven jumping probabilities $p_{i j}(\tau)$. (b)-(g) show the transition probabilities $p_{i j}(\tau)$ (represented as grey bars) that are fit by the von-Mises functional form, Eq. (9), (black solid lines) for north going (left column) and south going (right column) birds obtained from the satellite telemetry data.

rather than ring recovery data, fulfil these demands relatively well. Using telemetry data one can follow each individual bird's trajectory on its migratory path to estimate the transition times between predetermined resting, breeding or wintering places for each year.

In an exemplary way we have calculated a rather crude network from the satellite telemetry data of the three white stork individuals (Fig. 10). As the nodes of the network we defined the four main breeding and wintering areas of the species. There are surely additional resting areas used by the white stork $[40,43]$, however, here we want to keep the presentation as simple as possible. For each of the realised directed transitions we recorded the dates of leaving the one area and arriving in the other. The jumping times were defined as the mean time between the two events. By histogramming these data we were able to estimate the time-dependent flows $J_{i j}(t)=p_{i j}(\tau) n_{j}(t)$, from the model Eq. (7), between each pair of patches. After normalisation with the bird densities $n_{j}(t)$ this yields the desired transition probabilities $p_{i j}(\tau)$ (see Fig. 10, grey bars). We then, by hand, fitted a von-Mises functional form to these records, thus, having parametrised our model. Because of the low sample size we consider it not reasonable to use any more advanced fitting techniques, as e.g. maximum likelihood. They should be applied when larger data sets become available.

The transition probabilities in Fig. 10 provide valuable information about the spatio-temporal dynamics of the migration process. Despite the shortness of transition data all phases $\phi_{i j}$ have reasonably been reconstructed. Note that the phases $\phi_{i j}$ are ordered along the migratory path, in concord to the naive expectation. Further, the transitions are rather sharp and can be assumed to have the same width for all transitions. The estimated width equals $\sigma=0.12$ which corresponds to about 7 days. The small sample size does not allow a sensible estimation of the 
transition amplitudes, yet. Nevertheless, the crude fit in Fig. 10 appears already to be quite reasonable.

Interestingly, the transition amplitudes of the north going birds are all more or less equally of a large value. This reflects a basic north-south symmetry breaking in the migratory route of the white stork: In our data base the birds always return to their breeding area in the north (area 2 ), but in the southgoing direction their routes split into different resting and wintering areas. Thus, all north going transitions have a large amplitude $\left(h_{21} \approx h_{23} \approx h_{34}\right)$ which insures that all individuals jump within a short time interval. In contrast, when going south the birds have to decide on the western or eastern route, i.e. either to overwinter on the Iberian Penninsular (with probability $p_{12}$ ) or to fly to the Sudan (with probability $p_{32}$ ). Note that the transition to the Sudan area is slightly advanced $\phi_{32}<\phi_{12}$. Therefore, the transition probability $p_{32}$ has to be small, to ensure that some birds stay to use the western route, and $p_{12}$ must be large, so that no birds remain in Middle Europe over the winter. In this way we can explain the observed relation of the transition amplitudes $h_{32}<h_{12}$. A second branching arises in the Sudan region (area 3) where the birds have to decide whether to continue their migratory path to South Africa (area 4) or to stay. Consequently, the transition amplitude $h_{43}$ is rather small. This simple example already shows that it is not possible to read off the branching ratios simply from the transition parameters, if the transitions are not synchronised in season. Therefore, we expect that the seasonally driven model has nontrivial properties, especially for more complex network topologies.

As one can also observe for the white stork many migratory bird species exhibit differential migration [52]. This means that different populations, age groups or sexes of a species migrate on different routes, by varying time schedules and maybe even to different areas. Depending on how pronounced this effect is in the considered species a large amount of data is needed to obtain a sufficient picture of the species' migration patterns. The sample size here is rather small but well selected, so that we are confident that our model gives a good first approximation of the general migratory pattern of the white stork.

In principle one could also use ring data for parametrisation of the presented network migration model. However, as has already been pointed out, the major shortcoming of ring data is the difference in observation effort and reporting. For quantitative estimation (that we attempt here) the reporting rates would need to be quantified. We expect them to correlate with human population density or social indices such as alphabetisation or economic income, but any such relationship may also be illusory. Furthermore, ring data provide only the number of birds that have visited certain places during the year, but not exact time instances of the transitions between consecutive resting places. Therefore, jumping probabilities would have to be estimated from the observed densities by optimisation techniques like e.g. linear programming or simulated annealing. Hence, the use of ring data for estimating the transition probabilities of our bird migration model in time and space is possible, but poses many problems and it may render more successful to give other kinds of data the preference.

\section{Conclusions}

We strongly agree with and want to promote the notion of Bairlein [13] that the analysis of recovery data and modelling of migration patterns in time and space need increased emphasis in ecological research. On the one hand large amounts of bird observational data are collected on an ever increasing rate and quality. To gain insight into patterns and characteristics of such data they have to be analysed in much detail using statistical methods. On the other hand several research groups specialise on only few species and step by step try to disentangle its behaviour and ecology. As this is undoubtedly important we think it, however, crucial not to lose the more general view on ecology and e.g. bird migration mechanisms. Here the development of time and space explicit models may be of high value. With sensibly constructed models one could examine the influence of parameters as for example the peak time or interval length of leaving the breeding areas on the migration process. One could thus make predictions about the consequences of global factors such as climate change and its effects on the migration 
behaviour of several species and by simulations and model exploration obtain general insight into the underlying mechanisms. In the long run it may even be possible to make comparisons with the movement of other organism types.

The above described network model of bird migration makes many simplifying assumptions. In particular, it disregards any variability of the migration process between years. This means that environmental factors are assumed to have no influence on survival, timing and the course of migration. We are certainly aware that this is not the case in reality (e.g. $[53,54]$ ), especially in the light of the recent climate change. In this sense the presented ideas represent only a first step in the direction of setting up a full-fletched, conceptional bird migration model. Once the general properties of such a model are fully understood, other environmental covariates can be incorporated.

In this article we have summarised the kind of data that are available for quantitative studies of bird migration. Our aim is to encourage the systematic, theoretical study of this process, but also to call for caution regarding the use of such data. Of course, any kind of data needs to be carefully considered before it can be incorporated into a model. In bird migration we face the situation that much data has already been collected, and the advantages and disadvantages of them have to be taken into account before any decision can be made regarding its analysis. In theoretical modelling it is always possible to just explore a model and its properties per se, but only by parametrisation with sensible data one will be able to conclude sensible information about the ecological processes acting in avian migration.

We are grateful to the ringing centre Vogelwarte Hiddensee in Stralsund and the Vogelwarte Radolfzell at the Max-Plank-Institute for Ornithology for providing us with ring recovery and satellite telemetry data that was collected by numerous professional and amateur ornithologists for many years. We want to thank Ulrich Köppen and Birgit Gerkmann for discussing the data analysis and results. Special thanks to Michael Kaatz for elaborate discussions about data quality and Ralf Tönjes for help with model formulation. This work was funded by the VW-Stiftung and the BMBF.

\section{References}

1. P. Berthold, Vogelzug. Eine aktuelle Gesamtübersicht (Wissenschaftliche Buchgesellschaft, Darmstadt, 2000)

2. E. Schüz, P. Berthold, E. Gwinner, H. Oelke, Grundriß der Vogelzugkunde (Parey, Berlin, 1971)

3. R. Albert, A.-L. Barabási, Rev. Mod. Phys. 74, 47 (2002)

4. M.E.J. Newman, SIAM Rev. 45, 167 (2003)

5. D. Tilman, P. Kareiva, Spatial Ecology (Princeton University Press, Princeton, 1997)

6. R. Pastor-Satorras, A. Vespignani, Phys. Rev. Lett. 86, 3200 (2001)

7. T. Gross, C.J. Dommar, B. Blasius, Phys. Rev. Lett. 96, 208701 (2006)

8. L. Hufnagel, D. Brockmann, T. Geisel, PNAS 101, 15124 (2004)

9. G.-R. Walther, E. Post, P. Convey, A. Menzel, C. Parmesan, T.J.C. Beebee, J.-M. Fromentin, O. Hoegh-Guldberg, F. Bairlein, Nature 416, 389 (2002)

10. L. Jenni, M. Kéry, Proc. R. Soc. Lond. B 270, 1467 (2003)

11. Z. Hubalek, J. Wildlife, Diseases 40, 639 (2004)

12. A.M. Kilpatrick, A.A. Chmura, D.W. Gibbons, R.C. Fleischer, P.P. Marra, P. Daszak, PNAS 103, 19368 (2007)

13. F. Bairlein, Bird Study 50, 243 (2003)

14. B. Erni, F. Liechti, B. Bruderer, J. Theor. Biol. 219, 479 (2002)

15. A. Hedenström, T. Alerstam, J. Theor. Biol. 189, 227 (1997)

16. R. Wiltschko, W. Wiltschko, Animal Behav. 65, 257 (2003)

17. H. Mouritsen, Animal Behav. 56, 899 (1998)

18. T. Alerstam, Science 313, 791 (2006)

19. G.M. Viswanathan, V. Afanasyev, S.V. Buldyrev, E.J. Murphy, P.A. Prince, H.E. Stanley, Nature 381, 413 (1996)

20. H.W. Hethcote, SIAM Rev. 42, 599653 (2000)

21. B. Blasius, A. Huppert, L. Stone, Nature 399, 354 (1999)

22. F. Bairlein, Ardea 89, 7 (2001) 
23. W. Fiedler, F. Bairlein, U. Köppen, Advances in Ecological Research. Birds and Climate Change, edited by H. Caswell, Vol. 35 (Elsevier, Amsterdam, 2004), pp. 49-67

24. I.G. Priede, S.M. Swift (eds.), Wildlife Telemetry: Remote Monitoring and Tracking of Animals (Ellis Horwood Ltd., New York, 1992)

25. P. Berthold, E. Nowak, U. Querner, Falke 44, 134 (1997)

26. P. Jouventin, H. Weimerskirch, Nature 343, 746 (1990)

27. P. Berthold, W. van den Bossche, W. Fiedler, E. Gorney, M. Kaatz, Y. Leshem, E. Nowak, U. Querner, J. Ornithol. 142, 73 (2001)

28. K. Thorup, T. Alerstam, M. Hake, N. Kjellén, Oikos 103, 350 (2003)

29. W. van den Bossche, P. Berthold, M. Kaatz, E. Nowak, U. Querner, BfN-Skripten 66, 1 (2002)

30. A. Fukuda, M. Katsuji, E. Hirano, M. Suzuki, H. Higuchi, E. Morishita, D.J. Anderson, S. Waugh, R. Phillips, Mem. Natl. Inst. Polar Res. 58, 234 (2004)

31. M. Kaatz, Der Zug des Weißstorchs Ciconia ciconia auf der Europäischen Ostroute über den Nahen Osten nach Afrika, Ph.D. thesis (Halle/Saale, 2004)

32. M. Kaatz, Studie zur Neubewertung von ARGOS-Satellitenkoordinaten (1999) (unpublished)

33. A. Leick, GPS Satellite Surveying (John Wiley and Sons, New York, 1995)

34. H. Weimerskirch, F. Bonadonna, F. Bailleul, G. Mabille, G. Dell'Omo, H.-P. Lipp, Science 295 , $1259(2002)$

35. R.D. Hill, in Elephant Seals, Population, Ecology, Behaviour and Physiology, edited by B.J. Le Boeuf, R.M. Laws (Univ. of California Press, Berkeley, 1994), pp. 227-236

36. R.P. Wilson, Ardea 89, 231 (2001)

37. Y. Leshem, Y. Yom-Tov, Ibis 138, 188 (1996)

38. M. Wikelski, R.W. Kays, N.J. Kasdin, K. Thorup, J.A. Smith, G.W. Swenson Jr., J. Exper. Biol. 210, $181(2007)$

39. H.-G. Bauer, E. Bezzel, W. Fiedler, Das Kompendium der Vögel Mitteleuropas (Aula, Wiebelsheim, 2005)

40. P. Berthold, W. van den Bossche, M. Kaatz, U. Querner, Acta Zoo. Sin. 52, S211 (2006)

41. U. Köppen, S. Scheil, Apus 12, 5 (2004)

42. P. Berthold, M. Kaatz, U. Querner, J. Ornithol. 145, 356 (2004)

43. B. Gerkmann, Nutzung von Telemetrie- und Satellietendaten zur Identifizierung wichtiger Habitate wandernder Vogelarten, Ph.D. thesis (Bonn, 2007)

44. G. Creutz, Der Weißstorch. Ciconia Ciconia (A. Ziemsen Verlag, Wittenberg Lutherstadt, 1985)

45. N. Komin, U. Erdmann, L. Schimansky-Geier, Fluct. Noise Lett. 4, L151 (2004)

46. Z. Pasternak, B. Blasius, A. Abelson, J. Plankt. Research 26, 487 (2004)

47. R. Gutenkunst, M. Lutcavage, L. Edelstein-Keshet, J. Theor. Biol. 245, 243 (2007)

48. H. Bailey, P. Thompson, J. Animal Ecol. 75, 456 (2006)

49. J.M. Morales, D.T. Haydon, J. Frair, K.E. Holsinger, J.M. Fryxell, Ecology 85, 2436 (2004)

50. B.A. Nolet, W.M. Mooij, J. Animal Ecol. 71, 451 (2002)

51. P. Turchin, Quantitative Analysis of Movement: Measuring and Modeling Population Redistribution in Animals and Plants (Sinauer Associates, Sunderland, Massachusetts, 1998)

52. S.A. Gauthreaux, Avian Biol. 6, 93 (1982)

53. G. Zink, F. Bairlein, Der Zug europäischer Singvögel. Ein Atlas der Wiederfunde beringter Vögel (Aula, Wiesbaden, 1995)

54. A. Kölzsch, S.A. Sæther, H. Gustafsson, P. Fiske, J. Höglund, J.A. Kålås, J. Animal Ecol. 76, 740 (2007) 